\title{
Characteristics of spontaneous coagulase- negative staphylococcal spondylodiscitis: a retrospective comparative study versus Staphylococcus aureus spondylodiscitis
}

Julien Lopez ${ }^{1}$, Zuzana Tatar ${ }^{1}$, Anne Tournadre ${ }^{1}$, Marion Couderc ${ }^{1}$, Bruno Pereira², Martin Soubrier ${ }^{1}$ and Jean-Jacques Dubost ${ }^{1 *}$ (D)

\begin{abstract}
Background: Coagulase-negative staphylococci (CoNS) are increasingly implicated in recent patient series of spondylodiscitis, but there are no series of CoNS-spondylodiscitis available. The objective of this study was to compare the characteristics of patients with spontaneous CoNS-spondylodiscitis with those patients with Staphylococcus aureus (SA) spondylodiscitis.
\end{abstract}

Methods: This was a retrospective single center study involving 147 spontaneous infectious spondylodiscitis cases observed between 2000 and 2015. The 26 cases of CoNS-spondylodiscitis (15 confirmed) were compared with 30 cases of SA-spondylodiscitis. CoNS infection was considered confirmed if the same CoNS was isolated in at least two samples at two different times.

Result: Patients with CoNS-spondylodiscitis were older (70 vs. 61 years of age; $p=0.01$ ), had associated cancer more often (15\% vs. $0 \% ; p=0.04$ ) and had a longer diagnostic delay (>15 days in $88 \%$ vs. $60 \% ; p=0.01$ ); experienced fever less often (19\% vs. 50\%; $p=0.01$ ), and had lower white blood cell (7.6 vs. 9.9G/L; $p=0.01$ ) and polymorphonuclear leucocyte counts (5.6 vs. 7.5G/L; $p=0.04$ ). Patients with CoNS spondylodiscitis had less pronounced inflammatory syndrome (erythrocyte sedimentation rate [ESR]: $62 \mathrm{vs.} 81 \mathrm{~mm}$ at $1 \mathrm{~h} ; p=0.03 ;$ CRP: $60 \mathrm{vs} .147 \mathrm{mg} / \mathrm{L} ; p=0.0003$ ) and less common (ESR < $30 \mathrm{~mm}: 23 \%$ vs. $0 \% ; p=0.01 ; \mathrm{CRP}<10 \mathrm{mg} / \mathrm{L}: 23 \%$ vs. $0 \% ; p=0.005$ ) in comparison with patients with SA infection. The infection entry site was most often an intravascular catheter ( $20 \% \mathrm{vs} .3 \% ; p=0.008)$. The level of positive percutaneous needle biopsies was comparable between CoNS and SA. Two patients who died both had SA infections.

Conclusion: CoNS-spondylodiscitis involved at least 10\% of spontaneous spondylodiscitis cases and was more common in elderly patients, afflicted by comorbidities, and its presentation was less virulent than that of those with SA-spondylodiscitis.

Keywords: Infectious spondylodiscitis, Vertebral osteomyelitis, Coagulase-negative staphylococcus, Staphylococcus aureus

\footnotetext{
* Correspondence: jjdubost@chu-clermontferrand.fr

'Department of Rheumatology, University Hospital Gabriel Montpied, 58 rue

Montalembert, 63003 Clermont Ferrand CEDEX 1, France

Full list of author information is available at the end of the article
} 


\section{Background}

Infectious spondylodiscitis cases, like other osteoarticular infections, are mainly due to Staphylococcus aureus (SA). Coagulase-negative staphylococci (CoNS) have been implicated in patients with postoperative infectious spondylodiscitis. Isolated observations of spontaneous spondylodiscitis, primarily involving frail and immunosuppressed patients, are available in the literature [1,2]. In the spontaneous spondylodiscitis series published, the frequency of CoNS ranged from $3 \%$ to $11 \%$ [3-8], with increasing involvement reported in the most recent series [3, 9-12]. CoNS infections pose a problem in terms of accountability: it is often difficult to confirm that it is responsible for the infection and not just present as contamination.

In order to further assess the frequency and characteristics of spontaneous CoNS-spondylodiscitis cases, we reviewed the medical files in the rheumatology department since 2000 of all patients who were hospitalized for infectious spondylodiscitis. The features of patients with CoNS-infections were compared to those with $S$ aureus infections.

\section{Methods}

This was a retrospective single center study focused on the medical files of patients hospitalized for infectious spondylodiscitis over the period from January 2000 to May 2015. The rheumatology department of ClermontFerrand University Hospital, France, is a tertiary regional care center. Our department admits patients suffering from osteoarticular as well as spine diseases. The patients are admitted directly, or via the emergency or other departments, though they may also be transferred from other hospitals. Postoperative and tuberculosis infections were excluded from the analysis. The diagnosis was considered confirmed when a radiologist with significant experience in osteoarticular pathology thought that the imaging examinations, particularly the magnetic resonance imaging, were suggestive of infectious spondylodiscitis. These results were considered, along with compatible clinical and laboratory presentations, in addition to the patient's outcome after antibiotic therapy.

The data collected comprised demographic characteristics, infection site, microorganism type, identification method, initial clinical characteristics, risk factors including concomitant cancer, immunosuppressive treatment, ongoing corticoids therapy, diabetes, kidney insufficiency with renal clearance $<30 \mathrm{~mL} / \mathrm{min}$ or ongoing dialysis, drug addiction, severe liver disease, entry site (if identified), and laboratory parameters upon admission.

CoNS infection was considered confirmed if the same CoNS was isolated from two different samples (blood cultures, disco-vertebral biopsy, or another internal site) taken at different times. CoNS infection was considered probable if only one sample was positive.
Based on the data collected, comparisons were made between patients with CoNS-spondylodiscitis and those with SA-spondylodiscitis.

\section{Statistical analyses}

Statistical analyses were performed using Stata software (version 13, StataCorp, College Station, TX.US). All statistical tests were two-tailed with a type I error at 5\%. Continuous data were expressed as mean and 95\% confidence interval (95\% CI), depending on the statistical distribution, with an assumption of normality studied by the Shapiro-Wilk test. For quantitative data, the comparisons between groups (SA and CoNS, and confirmed and probable CoNS cases) were carried out using either Student's t-test or Mann-Whitney test if the t-test conditions were not met ([i] normality and [ii] homoscedasticity studied by the Fisher-Snedecor test). For categorical parameters, the between-group comparisons were performed using Chi-squared test or Fisher's exact test, as appropriate.

\section{Results}

Between January 2000 and May 2015, a total of 147 patients were hospitalized in the rheumatology department for non-tuberculosis spontaneous infectious spondylodiscitis. Among these, 26 cases were due to CoNS, with the diagnosis confirmed in 15 and were considered probable in 11 cases (Table 1); 30 cases were due to SA. Overall, CoNS-spondylodiscitis cases involved $10 \%$ to $18 \%$ of the spontaneous infections according to the diagnostic criteria used. CoNS comprised $S$. epidermidis $(n=20), S$. simulans $(n=1), S$. warneri $(\mathrm{n}=1)$, S. lugdunensis $(\mathrm{n}=1), S$. hominis $(\mathrm{n}=1)$, S. haemolyticus $(\mathrm{n}=1)$, and unclassified $(\mathrm{n}=1)$.

\section{Comparison between CoNS-spondylodiscitis and SA- spondylodiscitis cases}

The male predominance was similar for CoNS and SA infections (73 vs. 70\%; $\mathrm{p}=\mathrm{NS}$ ) (Table 2). Patients with CoNS infection were older (70 vs. 61 years; $p=0.01$ ) and had associated cancer more often (15 vs. $0 \%$; $p=0.04)$. The frequency of diabetes in both was comparable. There were no differences in spondylodiscitis sites between the two groups. In patients infected with CoNS, there was a longer diagnostic delay than in those infected with SA (48 vs. 24 days; $p=0.004$ ). This delay exceeded 15 days in $88 \%$ of patients with CoNS vs. $60 \%$ of patients with SA $(p=0.01)$. The CoNS infections were less often accompanied by fever (19\% vs. $50 \%$; $p=0.01$ ), and with lower white blood cell (7.6 vs. 9.9G/ $\mathrm{L} ; p=0.01$ ) and polymorphonuclear leucocyte (5.6 vs. $7.5 \mathrm{G} / \mathrm{L} ; p=0.04)$ counts. Inflammatory syndrome was less pronounced (erythrocyte sedimentation rate [ESR]: 62 vs. $81 \mathrm{~mm}$ at $1 \mathrm{~h} ; p=0.03$; C-reactive protein [CRP]: 
Table 1 Bacteriological characteristics of patients with CoNS spondylodiscitis

\begin{tabular}{|c|c|c|c|c|c|c|c|c|}
\hline \multirow[t]{2}{*}{ Cases } & \multirow[t]{2}{*}{ Microorganism } & \multicolumn{3}{|c|}{ Percutaneous needle biopsy } & \multicolumn{2}{|l|}{ Blood cultures } & \multirow[t]{2}{*}{ Others } & \multirow[t]{2}{*}{ Probability } \\
\hline & & Performed Y/N & Positive $\mathrm{Y} / \mathrm{N}$ & $\begin{array}{l}\text { Number of } \\
\text { media }\end{array}$ & $\begin{array}{l}\text { Number of blood cultures } \\
\text { positive/ Number performed }\end{array}$ & $\begin{array}{l}\text { Number of } \\
\text { media }\end{array}$ & & \\
\hline 1 & S. epidermidis & $\mathrm{Y}$ & $\mathrm{Y}$ & 1 & $0 / 10$ & 0 & $\mathrm{~N}$ & probable \\
\hline 2 & S. epidermidis & Y & Y & 3 & $0 / 20$ & 0 & IV device & confirmed \\
\hline 3 & S.haemolyticus & N & / & / & $4 / 12$ & 6 & $\mathrm{~N}$ & confirmed \\
\hline 4 & S. epidermidis & Y & $\mathrm{N}$ & 0 & $5 / 10$ & 9 & IV device & confirmed \\
\hline 5 & S. epidermidis & Y & Y & 2 & $2 / 14$ & 2 & $\mathrm{~N}$ & confirmed \\
\hline 6 & S. epidermidis & Y & Y & 3 & $0 / 5$ & 0 & N & probable \\
\hline 7 & S. epidermidis & Y & Y & 3 & $0 / 9$ & 0 & N & probable \\
\hline 8 & S.warneri & Y & Y & 1 & $0 / 2$ & 0 & N & probable \\
\hline 9 & S. epidermidis & $Y^{a}$ & Y & 3 & $0 / 16$ & 0 & N & confirmed \\
\hline 10 & S. epidermidis & $\mathrm{N}$ & / & / & $6 / 6$ & 12 & $\mathrm{~N}$ & confirmed \\
\hline 11 & S. epidermidis & Y & Y & 2 & $4 / 14$ & 7 & IV device & confirmed \\
\hline 12 & S. epidermidis & Y & Y & 2 & $0 / 5$ & 0 & N & probable \\
\hline 13 & S.hominis & Y & $\mathrm{N}$ & 0 & $1 / 8$ & 1 & N & probable \\
\hline 14 & S.lugdunensis & N & / & / & $0 / 6$ & 0 & 3 knee punctures & confirmed \\
\hline 15 & S.simulans & $\mathrm{N}$ & / & / & $3 / 3$ & 3 & $\mathrm{~N}$ & confirmed \\
\hline 16 & S. epidermidis & Y & Y & 2 & $0 / 9$ & 0 & $\mathrm{~N}$ & probable \\
\hline 17 & S.coagulase negative & Y & Y & 1 & $1 / 11$ & 1 & $\mathrm{~N}$ & confirmed \\
\hline 18 & S. epidermidis & Y & Y & 3 & $5 / 5$ & 6 & $\mathrm{~N}$ & confirmed \\
\hline 19 & S. epidermidis & Y & Y & 1 & $0 / 17$ & 0 & $\mathrm{~N}$ & probable \\
\hline 20 & S. epidermidis & $N$ & / & / & $6 / 8$ & 11 & $\mathrm{~N}$ & confirmed \\
\hline 21 & S. epidermidis & Y & Y & 1 & $0 / 11$ & 0 & $\mathrm{~N}$ & probable \\
\hline 22 & S. epidermidis & Y & Y & 1 & $0 / 6$ & 0 & $\mathrm{~N}$ & probable \\
\hline 23 & S. epidermidis & Y & Y & 1 & $1 / 3$ & 2 & $\mathrm{~N}$ & confirmed \\
\hline 24 & S. epidermidis & $N$ & / & / & $2 / 10$ & 3 & $\mathrm{~N}$ & confirmed \\
\hline 25 & S. epidermidis & N & / & / & $0 / 11$ & 0 & 1 hip puncture & probable \\
\hline 26 & S.epidermidis & Y & $\mathrm{N}$ & 0 & $3 / 8$ & 3 & $\mathrm{~N}$ & confirmed \\
\hline
\end{tabular}

Confirmed: CoNS were isolated from at least two different samples taken at different times. Probable: only one sample was positive

${ }^{\text {aTwo }}$ percutaneous needle biopsy performed

60 vs. $147 \mathrm{mg} / \mathrm{L} ; p=0.0003)$ and less common $(\mathrm{ESR}<30 \mathrm{~mm}: 23 \%$ vs. $0 \% ; \mathrm{p}=0.01 ; \mathrm{CRP}<10 \mathrm{mg} / \mathrm{L}$ : $23 \%$ vs. $0 \% ; p=0.005)$ compared to patients with SA infections. The entry site was an intravascular catheter more often (20 vs. $3 \% ; p=0.08$ ). Blood cultures tended to be positive less often in those with CoNS infections ( $50 \%$ vs. $71 \%$; $p=0.1$ ), with percutaneous needle biopsies performed more often ( $73 \%$ vs. $37 \%$; $p=0.008)$, yet with comparable positivity rates for CoNS and SA infection cases (84 vs. $82 \%$; $\mathrm{p}=\mathrm{NS}$ ). Overall, CoNS infections in 10 patients (38\%) and SA infections in six patients (20\%) were methicillin-resistant $(p=N S)$. The duration of the antibiotic therapy did not differ between the two groups, yet SA-spondylodiscitis cases had a longer hospital stay. It should be noted that the two patients who died were both infected with SA.

\section{Comparison of confirmed CoNS-spondylodiscitis and SA-spondylodiscitis cases}

Patients with confirmed CoNS infections were older than those with SA infections (Table 2) and had associated cancer more often ( $20 \%$ vs. $0 \% ; p=0.04)$. Additionally, inflammatory syndrome was less pronounced (CRP: 76 vs. $147 \mathrm{mg} / \mathrm{L} ; p=0.02$ ), with CRP $<10 \mathrm{mg} / \mathrm{L}$ in $20 \%$ of CoNS infection cases versus CRP $>10 \mathrm{mg} / \mathrm{L}$ in all $\mathrm{SA}$ infection cases. Antibiotic therapy duration was shorter in patients with confirmed CoNS spondylodiscitis versus those with SA spondylodiscitis (75 vs. 91 days; $p=0.05$ ).

\section{Discussion}

In our cohort of spontaneous spondylodiscitis cases, at least $10 \%$ were caused by CoNS infections. Compared to SA spondylodiscitis cases, CoNS spondylodiscitis affected 
Table 2 Comparison of characteristics of patients with spondylodiscitis caused by coagulase-negative staphylococcus (CoNS) and caused by Staphylococcus aureus

\begin{tabular}{|c|c|c|c|c|c|c|c|}
\hline & $\begin{array}{l}\text { 1: S.aureus } \\
n=30\end{array}$ & $\begin{array}{l}\text { 2: CoNS } \\
\text { total } \\
n=26\end{array}$ & $\begin{array}{l}\text { 3: CoNS } \\
\text { confirmed } \\
n=15\end{array}$ & $\begin{array}{l}\text { 4:CoNS } \\
\text { probable } \\
n=11\end{array}$ & $\begin{array}{l}p \\
1 \text { vs } 2\end{array}$ & $\begin{array}{l}p \\
1 \text { vs } 3\end{array}$ & $\begin{array}{l}p \\
3 \text { vs } 4\end{array}$ \\
\hline Age (mean [95\% Cl]) & $61[56 ; 67]$ & $70[66 ; 75]$ & $71[64 ; 78]$ & $70[64 ; 76]$ & 0.01 & 0.02 & 0.6 \\
\hline Male $n(\%)$ & $21(70)$ & $19(73)$ & $11(73)$ & $8(73)$ & 0.8 & 0.8 & 1 \\
\hline Site $n(\%)$ & & & & & 0.7 & 0.8 & 0.3 \\
\hline - Cervical & $3(10)$ & $3(12)$ & $1(7)$ & $2(18)$ & & & \\
\hline - Thoracic & $7(23)$ & $7(27)$ & $3(20)$ & $4(36)$ & & & \\
\hline - Lumbar & $18(60)$ & $16(62)$ & $11(73)$ & $5(45)$ & & & \\
\hline $\begin{array}{l}\text { Symptoms for more than } \\
15 \text { days } n(\%)\end{array}$ & $18(60)$ & $23(88)$ & $13(87)$ & $10(91)$ & 0.01 & 0.07 & 0.7 \\
\hline $\begin{array}{l}\text { Duration (d) of hospitalization } \\
\text { (median }[95 \% \mathrm{Cl}] \text { ) }\end{array}$ & $28[23.4 ; .32 .6]$ & $21.5[16.5 ; 26.4]$ & $23.6[15.8 ; 31.4]$ & $18.5[12.4 ; 24.7]$ & 0.05 & 0.3 & 0.3 \\
\hline Temperature $>38^{\circ} \mathrm{C} \mathrm{n}(\%)$ & $15(50)$ & $5(19)$ & $5(33)$ & $0(0)$ & 0.01 & 0.3 & 0.05 \\
\hline Presence of risk factors n (\%) & $14(47)$ & $15(58)$ & $9(60)$ & $6(55)$ & 0.4 & 0.4 & 0.8 \\
\hline Cancer $n(\%)$ & $0(0)$ & $4(15)$ & $3(20)$ & $1(9)$ & 0.04 & 0.03 & 0.6 \\
\hline Diabetes $n(\%)$ & $9(30)$ & $8(31)$ & $5(33)$ & $3(27)$ & 0.9 & 0.8 & 1 \\
\hline \multicolumn{8}{|l|}{ Entry site } \\
\hline -Cutaneous n (\%) & $9(30)$ & $6(23)$ & $2(13)$ & $4(36)$ & 0.5 & 0.3 & 0.13 \\
\hline -Catheter $n(\%)$ & $1(3)$ & $5(19)$ & $3(20)$ & $2(18)$ & 0.08 & 0.1 & 1 \\
\hline Blood cultures n (\%) & $20(71)$ & $13(50)$ & $12(80)$ & $1(9)$ & 0.1 & 0.5 & 0.001 \\
\hline $\begin{array}{l}\text { Percutaneous needle biopsy } \\
\text { made } \mathrm{n}(\%)\end{array}$ & $11(37)$ & $19(73)$ & $9(60)$ & $10(90)$ & 0.008 & 0.1 & 0.07 \\
\hline $\begin{array}{l}\text { Positive percutaneous needle } \\
\text { biopsy n (\%) }\end{array}$ & $9(82)$ & $16(84)$ & $7(78)$ & $9(90)$ & 0.8 & 0.8 & 0.46 \\
\hline $\begin{array}{l}\text { Leukocytes (G/L) (mean } \\
[95 \% \mathrm{Cl}])\end{array}$ & $9.9[8.4 ; 11.4]$ & $7.6[6.6 ; 8.6]$ & $8.3[6.8 ; 9.7]$ & $6.7[5.2 ; 8.3]$ & 0.01 & 0.5 & 0.1 \\
\hline PMN (G/L) (mean $[95 \%$ CI]) & $7.5[6.0 ; 9.0]$ & $5.6[4.6 ; 6.6]$ & $6.3[4.9 ; 7.7]$ & $4.6[3.2 ; 6.0]$ & 0.04 & 0.1 & 0.07 \\
\hline $\mathrm{ESR}<30$ at $1 \mathrm{~h} n(\%)$ & $0(0)$ & $5(23)$ & $2(15)$ & $3(33)$ & 0.01 & 0.1 & 0.3 \\
\hline CRP > 10 mg/L n (\%) & $30(100)$ & $20(77)$ & $12(80)$ & $8(73)$ & 0.005 & 0.02 & 0.6 \\
\hline $\begin{array}{l}\text { Antibiotic therapy duration (d) } \\
\text { (median }[95 \% \text { CI]) }\end{array}$ & $91.5[84.4 ; 98.7]$ & $82.6[74.5 ; 90.6]$ & $75.6[63.7 ; 87.5]$ & $93.0[86.2 ; 99.8]$ & 0.1 & 0.05 & 0.09 \\
\hline Sensitivity to methicillin $n(\%)$ & $24(80)$ & $16(61)$ & $10(67)$ & $6(55)$ & 0.1 & 0.2 & 0.7 \\
\hline
\end{tabular}

$P M N$ polymorphonuclear leucocytes, ESR erythrocyte sedimentation rate, CRP C-reactive protein

older patients, with concomitant cancer and who exhibited less virulent infection symptoms. One out of 5 entry sites was an intravascular catheter.

Over recent years, CoNS infections have been shown to be increasing in frequency [13]. Most often, these infections are of nosocomial origin and are associated with foreign object implantations, mostly prostheses and catheters [14]. The main difficulty consists of confirming the microorganism responsible, given that there is no reliable tool to differentiate between infection and contamination, though most authors deem infection to be highly likely when the same microorganism is isolated in at least two samplings taken at two different times [13, 15].

The frequency of CoNS spondylodiscitis was shown to vary greatly among the series published in the literature, depending mostly on the inclusion criteria. The frequency rates vary from $3 \%$ to $11 \%$ in spontaneous spondylodiscitis series [3-8]. In a recent French multicenter study on spontaneous spondylodiscitis with negative blood cultures, CoNS was the microorganism most frequently identified by percutaneous needle biopsy, representing $18 \%$ of the spondylodiscitis (17/ 95) and $61 \%(17 / 28)$ of the staphylococcal spondylodiscitis cases [16].This microorganism has been increasingly implicated in the most recent series, and Lora Tamayo et al. suggested that the higher frequency of these slightly virulent microorganism translates may be accounted for by epidemiological changes [3]. This may result from the increased age of the populations and comorbidities, particularly cancer, as seen in our study. 
Moreover, because catheters are a common portal entry for CoNS, hemodialysis has not surprisingly been reported as a relevant risk factor for CoNS spondylodiscitis [17]. The increase in the occurrence of CoNS spondylodiscitis likely reflects the increased infection rates caused by this microorganism, as noted over recent years [8].

CoNS infection is indolent and is characterized by largely non-specific symptoms and an attenuated infectious syndrome. Less than one-third of patients display fever. In one out of five cases in our study infectious syndrome was completely lacking accountings for the substantial diagnostic delay. The indolence appeared to be even more pronounced in patients with probable CoNS, although we cannot exclude contamination in some cases. The low frequency of positive blood cultures retrieved from this group can probably be explained, at least to some extent, by the inclusion criteria used.

Common resistance to antibiotics proves to be another characteristic of CoNS infection. In our cohort, a higher level of methicillin-resistant strains was found in CoNS cases, compared to SA cases, but the difference did not reach statistical significance, probably because the low patient numbers. The frequency of methicillin-resistant strains identified in our cohort was comparable to that revealed in osteoarticular infections in a French orthopedic department, with $23 \%$ of SA and $44 \%$ of CoNS cases found to be resistant to methicillin [18].

This study has several limitations, particularly its retrospective study design. In addition, because of its center design, extrapolation of our results to other centers requires great caution. Though this cohort is the largest of its kind, the number of patients with confirmed CoNS spondylodiscitis was relatively small, which may have limited the study's potential to detect differences with the SA spondylodiscitis group.

\section{Conclusion}

While waiting for reliable bacteriological techniques capable of differentiating between infection and contamination, identifying CoNS from a bacteriological examination must be considered for the diagnosis of spondylodiscitis, and a systematic conclusion on contamination should be avoided.

\section{Abbreviations}

CoNS: Coagulase-negative staphylococcal; CRP: C-reactive protein; ESR: Erythrocyte sedimentation rate; MRI: Magnetic resonance imaging; PMN: Polymorphonuclear leucocytes; SA: Staphylococcus aureus

\section{Acknowledgments}

Not applicable.

\section{Funding}

The authors have no support or funding to report.
Availability of data and materials

Data and supporting materials associated with this study will be provided upon request by the corresponding author.

\section{Authors' contributions}

JJD designed the study, was responsible for the statistical analysis and interpretation, and wrote the article. $\mathrm{JL}$ participated in the design of the study, the data collection, has been contributed to the analysis and interpretation of data, and has been involved in drafting the manuscript; ZT contributed to the data collection and has been involved in revising critically the manuscript for important intellectual content; AT contributed to the data collection and has been involved in revising critically the manuscript for important intellectual content. MC contributed to the data collection and has been involved in revising critically the manuscript for important intellectual content. BP contributed to the analysis and interpretation of data and has been involved in drafting the manuscript. MS participated in the design of the study, contributed to the data analysis and interpretation. All authors have read and approved the final version of the manuscript.

\section{Ethics approval and consent to participate}

The study protocol was approved by the ethical comitee of ClermontFerrand (CPP Sud Est VI). The ethics committee waived the need for written consent provided by participants due to the retrospective nature of the study.

Consent for publication

Not applicable.

\section{Competing interests}

The authors declare that they have no competing interests.

\section{Publisher's Note}

Springer Nature remains neutral with regard to jurisdictional claims in published maps and institutional affiliations.

\section{Author details}

1'Department of Rheumatology, University Hospital Gabriel Montpied, 58 rue Montalembert, 63003 Clermont Ferrand CEDEX 1, France. ${ }^{2}$ Biostatistics Unit, Clermont-Ferrand University Hospital, Clermont-Ferrand, France.

Received: 1 March 2017 Accepted: 3 October 2017

Published online: 13 October 2017

References

1. Isenberg Y, Parada JP. Spontaneous vertebral osteomyelitis due to Staphylococcus Epidermidis. J Med Microbiol. 2010;59:599-601.

2. Belzunegui J, Intxausti JJ, De Dios JR, et al. Haematogenous vertebral osteomyelitis caused by Staphylococcus Epidermidis: report of 4 cases. Clin Exp Rheumatol. 2000;18:115-6.

3. Lora-Tamayo J, Euba G, Narváez JA, et al. Changing trends in the epidemiology of pyogenic vertebral osteomyelitis: the impact of cases with no microbiologic diagnosis. Semin Arthritis Rheum. 2011:41:247-55.

4. Friedman JA, Maher CO, Quast LM, et al. Spontaneous disc space infections in adults. Surg Neurol. 2002;57:81-6.

5. Nolla JM, Ariza J, Gómez-Vaquero C, et al. Spontaneous pyogenic vertebral osteomyelitis in nondrug users. Semin Arthritis Rheum. 2002;31:271-8.

6. D'Agostino C, Scorzolini L, Massetti AP, et al. A seven-year prospective study on spondylodiscitis: epidemiological and microbiological features. Infection. 2010;38:102-7.

7. Bhavan KP, Marschall J, Olsen MA, et al. The epidemiology of hematogenous vertebral osteomyelitis: a cohort study in a tertiary care hospital. BMC Infect Dis. 2010;10:158

8. Kehrer M, Pedersen $C$, Jensen $T G$, et al. Increasing incidence of pyogenic spondylodiscitis: a 14-year population-based study. J Infect. 2014;68:313-20.

9. Aagaard T, Roed C, Dragsted C, et al. Microbiological and therapeutic challenges in infectious spondylodiscitis: a cohort study of 100 cases, 20062011. Scand J Infect Dis. 2013;45:417-24.

10. Mylona E, Samarkos M, Kakalou E, et al. Pyogenic vertebral osteomyelitis: a systematic review of clinical characteristics. Semin Arthritis Rheum. 2009;39:10-7.

11. Corrah TW, Enoch DA, Aliyu SH, et al. Bacteraemia and subsequent vertebral osteomyelitis: a retrospective review of 125 patients. QJM. 2011;104:201-7. 
12. Loibl M, Stoyanov L, Doenitz C, Brawanski A, et al. Outcome-related cofactors in 105 cases of vertebral osteomyelitis in a tertiary care hospital. Infection. 2014;42:503-10.

13. Pfaller MA, Herwaldt LA. Laboratory, clinical, and epidemiological aspects of coagulase-negative staphylococci. Clin Microbiol Rev. 1988;1:281-99.

14. Bucher E, Trampuz A, Donati L, et al. Spondylodiscitis associated with bacteraemia due to coagulase-negative staphylococci. Eur J Clin Microbiol Infect Dis. 2000;19:118-20.

15. Ahlstrand E, Bäckman A, Persson $L$, et al. Evaluation of a PCR method to determine the clinical significance of blood cultures with Staphylococcus Epidermidis in patients with hematological malignancies. APMIS. 2014;122:539-44.

16. Gras G, Buzele R, Parienti JJ, et al. Microbiological diagnosis of vertebral osteomyelitis: relevance of second percutaneous biopsy following initial negative biopsy and limited yield of post-biopsy blood cultures. Eur J Clin Microbiol Infect Dis. 2014;33:371-5.

17. Helewa RM, Embil JM, Boughen CG, Cheang M, Goytan M, Zacharias JM, Trepman E. Risk factors for infectious spondylodiscitis in patients receiving hemodialysis. Infect Control Hosp Epidemiol. 2008;29:567-71.

18. Titécat M, Senneville $E$, Wallet F, et al. Bacterial epidemiology of osteoarticular infections in a referent center: 10-year study. Orthop Traumatol Surg Res. 2013;99:653-8.

\section{Submit your next manuscript to BioMed Central and we will help you at every step:}

- We accept pre-submission inquiries

- Our selector tool helps you to find the most relevant journal

- We provide round the clock customer support

- Convenient online submission

- Thorough peer review

- Inclusion in PubMed and all major indexing services

- Maximum visibility for your research

Submit your manuscript at www.biomedcentral.com/submit 